\title{
Sweeping surfaces with Natural mate curve of a spatial curve in Euclidean 3-Space
}

\author{
Rashad A. Abdel-Baky ${ }^{a}$, and Monia F. Naghi ${ }^{b}$ \\ ${ }^{a}$ Department of Mathematics, Faculty of Science, University of Assiut, Assiut 71516, Egypt \\ ${ }^{b}$ Department of Mathematics, Faculty of Science, King Abdulaziz University, Jeddah, Saudi Arabia
}

Abstract: In this paper, we introduce the notion of sweeping surfaces with Natural mate curve of a spatial curve in Euclidean 3-space $E^{3}$. We also show that the parametric curves on these surfaces are lines of curvature. Then, we derive the necessary and sufficient condition for the sweeping surface to become a developable ruled surface. In particular, we analyze the necessary and sufficient conditions when the resulting developable surface is a cylinder, cone or tangent surface. Finally, some representative curves are chosen to construct the corresponding developable surfaces which possessing these curves as lines of curvature.

Key-Words: textbf Natural mate curve, Profile curve, Developable surface. MSC (2000). 53A04, 53A05, $53 \mathrm{~A} 17$.

Received: June 7, 2020. Revised: November 10, 2020. Accepted: November 26, 2020. Published: December 8, 2020.

\section{Introduction}

A canal or channel surface is a surface traced out as the envelope of a one-parameter family of sphereswhose centers lie on a space curve, its spine or directrix: When the radii of the generating spheres areconstant the canal surface is the envelope of a moving sphere and is called a sweeping or pipe surface.There are several examples that we are familiar with, such as: right circular cylinder (spine is a line, theaxis of the cylinder), torus (directrix is a circle), right circular cone (spine is a line (the axis), radii ofthe spheres not constant), and surface of revolution (spine is a line). This concept is a generalization ofthe classical notion of a mate of a planar curve [1-8.]

Sweeping surfaces play an essential rolein Computer Aided Geometric Design (CAGD),such as construction of robotic path planning,blending surfaces, transition surfaces betweenpipes, manufacturing of sculptured surfaces [8,9]. One of the important fact about sweeping surfaceis that the sweeping surface can be a developablesurface [1113]. Developable surfaces havea very important place in mathematics and engineeringsuch as motion analysis or designing carsand ships. There are three types of developableruled surfaces: cylinders, cones and tangent surfaces[13-16].
Several studies in the previous literature thatmay be inclusived in the area of our treatise areas follows: Do Carmo discussed some geometricalfeatures of pipe surfaces and proved two veryremarkable theorems, namely Fary\{Milnor theoremand Fenchel's theorem [1]. In [2], $\mathrm{Lu}$ andPottmann proved that a canal surface with a rationalspine curve constantly admits a rationalparametrization and o ered an algorithm for itscomputation. In [3], Stasiak and Maddocks modeledDNA molecules and parts of proteins as deformablecanal surfaces and classi ed the betterpacking problem. Maritan et al. [4] studied theproblem locating the optimal shapes of closelypacked canal surfaces. Krivoshapko, and Hyengstuided the geometric researches of cyclic surfaceswith generating circles of constant and several diameters[5]. Kim et al. [6] considered a particulartype of sweeping surface named canal surfaces inEuclidean 3 -space. For such a kind of surfaces,some interesting and important relations aboutthe Gaussian curvature, the mean curvature andthe second Gaussian curvature are derived. Qianet al. [7] studied canal surfaces in the Euclidean3-space in terms of their Gauss map, and obtaineda complete classi cation of canal surfaces whoseGauss maps are of the so-called pointwise 1type.Soliman et al. [8] investigated a particular Weingartenand linear Weingarten varieties of canalsurfaces according to Bishop frame in Euclidean3-space satisfying some fascinating and necessary 
equations in terms of the Gaussian curvature, the mean curvature.

In 1975, Bishop [17] introduced a new frame called the alternetive frame or Bishop frame, which could provide the desired means to slide along a space curve. It has become a useful tool for animations, motion planning, computer vision, and related applications where the SerretFrenet frame may prove unsuitable. For example, it may be use to compute the shape of sequences of DNA using a curve defined by the Bishop frame. The Bishop frame may be also produce a way to control virtual cameras in computer animations $[11,12,19]$.

In this paper, sweeping surfaces with Natural mate curve of a curve are proposed and further applied to surface modeling. By establishing the Natural rotation-minimizing (NRMF) of Natural mate curve of a spatial curve, we express parametric representation for a sweeping surface and study its local singularities and convexity. In terms of this, the necessary and sufficient condition for the sweeping surface to be a developable ruled surface is derived. Finally, we illustrate the convenience and efficiency of this method by some representative examples. This study is intended to conduct the geometric analysis of sweeping surfaces through the Natural mate curve of a spatial curve.

\section{Preliminaries}

The ambient space is the Euclidean 3 -space $E^{3}$, and for our work we have used [1,9] as general references. Let $\alpha=\alpha(s)$ be a unit speed curve in Euclidean 3 -space $R^{3}$; by $\kappa(s)$ and $\tau(s)$ we denote the natural curvature and torsion of $\alpha(s)$, respectively. Let $\{\mathbf{T}(s), \mathbf{N}(s), \mathbf{B}(s)\}$ be the SerretFrenet frame associated with the curve $\alpha(s)$, then the Serret-Frenet formulae read:

$$
\begin{gathered}
\left(\begin{array}{l}
\mathbf{T}^{\prime} \\
\mathbf{N}^{\prime} \\
\mathbf{B}^{\prime}
\end{array}\right)=\left(\begin{array}{lll}
0 & \kappa(s) & 0 \\
-\kappa(s) & 0 & \tau(s) \\
0 & -\tau(s) & 0
\end{array}\right)\left(\begin{array}{l}
\mathbf{T} \\
\mathbf{N} \\
\mathbf{B}
\end{array}\right) \\
\quad=\boldsymbol{\Omega} \times\left(\begin{array}{l}
\mathbf{T} \\
\mathbf{N} \\
\mathbf{B}
\end{array}\right),
\end{gathered}
$$

. where $\boldsymbol{\Omega}(s)=\tau \mathbf{T}+\kappa \mathbf{B}$ is the angular velocity of the Serret-Frenet frame. Here a dash denotes differentiation with respect to $s$.

Definition 2.1. Let curve $\beta\left(s_{n}\right)$ be the integral curve of the principal normal vector $\mathbf{N}(s)$ of $\alpha(s)$, that is $\beta\left(s_{n}\right)=0 s \int \mathbf{N}(s) d s$, the curve $\beta\left(s_{n}\right)$ is called the Natural mate curve of $\alpha(s)$, and the pair $\left\{\alpha(s), \beta\left(s_{n}\right)\right\}$ is called the Natural pair [20].

It can be also shown that the arc length parameter $s_{n}$ of the curve $\beta\left(s_{n}\right)$ can be expressed as $s_{n}=s+c$, where $\mathrm{c}$ is a constant, without losing of generality, we can take $c=0$, that is, $s=s_{n}$. The Serret-Frenet frame $\left\{\mathbf{T}_{n}(s), \mathbf{N}_{n}(s), \mathbf{B}_{n}(s)\right\}$ along $\beta(s)$ satisfies the following formula

$$
\left(\begin{array}{l}
\mathbf{T}_{n} \\
\mathbf{N}_{n} \\
\mathbf{B}_{n}
\end{array}\right)=\left(\begin{array}{lll}
0 & 1 & 0 \\
-\cos \psi & 0 & \sin \psi \\
\sin \psi & 0 & \cos \psi
\end{array}\right)\left(\begin{array}{l}
\mathbf{T} \\
\mathbf{N} \\
\mathbf{B}
\end{array}\right)
$$

where

$$
\cos \psi=\frac{\kappa}{\sqrt{\kappa^{2}+\tau^{2}}}, \quad \sin \psi=\frac{\tau}{\sqrt{\kappa^{2}+\tau^{2}}} .
$$

Furthermore, we have

$$
\begin{aligned}
& \left(\begin{array}{l}
\mathbf{T}_{n}^{\prime} \\
\mathbf{N}_{n}^{\prime} \\
\mathbf{B}_{n}^{\prime \prime}
\end{array}\right)=\left(\begin{array}{lll}
0 & \kappa_{n}(s) & 0 \\
-\kappa_{n}(s) & 0 & \tau_{n}(s) \\
0 & -\tau_{n}(s) & 0
\end{array}\right)\left(\begin{array}{l}
\mathbf{T}_{n} \\
\mathbf{N}_{n} \\
\mathbf{B}_{n}
\end{array}\right) \\
& =\boldsymbol{\Omega}_{n} \times\left(\begin{array}{c}
\mathbf{T}_{n} \\
\mathbf{N}_{n} \\
\mathbf{B}_{n}
\end{array}\right),
\end{aligned}
$$

where $\boldsymbol{\Omega}_{n}(s)=\tau_{n} \mathbf{T}_{n}+\kappa_{n} \mathbf{B}_{n}$ is the angular velocity of the Serret-Frenet frame $\left\{\mathbf{T}_{n}(s), \mathbf{N}_{n}(s)\right.$, $\left.\mathbf{B}_{n}(s)\right\}$. Here,

$$
\kappa_{n}(s)=\sqrt{\kappa^{2}+\tau^{2}}, \tau_{n}(s)=\frac{\kappa^{2}}{\kappa^{2}+\tau^{2}}\left(\frac{\tau}{\kappa}\right)^{\prime} .
$$

Definition 2.2. An orthonormal moving frame $\left\{\xi_{1}, \xi_{3}, \xi_{3}\right\}$, along a space curve $\gamma(s)$, is rotation minimizing frame (RMF) with respect to $\xi_{1}$ if its angular velocity $\omega$ satisfies $<\omega, \xi_{1}>=0$ or, equivalently, the derivatives of $\xi_{2}$ and $\xi_{3}$ are both parallel to $\xi_{1}$. An analogous characterization holds when $\xi_{2}$ or $\xi_{3}$ is chosen as the reference direction [19].

According to the Definition 2.2, we observe that the Serret-Frenet frame is rotationminimizing with respect to the principal normal $\mathbf{N}_{2}$, but not with respect to the tangent $\mathbf{T}_{n}$ and the binormal $\mathbf{B}_{n}$. Although the Serret-Frenet frame is not rotation minimizing with respect to $\mathbf{B}_{n}$, one can easily derive such a rotation minimizing frame from it. New normal plane vectors $\left(\mathbf{N}_{1}, \mathbf{N}_{2}\right)$ are specified through a rotation of $\left(\mathbf{N}_{n}, \mathbf{B}_{n}\right)$ according to

$$
\left(\begin{array}{l}
\mathbf{T}_{1} \\
\mathbf{N}_{1} \\
\mathbf{N}_{2}
\end{array}\right)=\left(\begin{array}{lll}
1 & 0 & 0 \\
0 & \cos \varphi & \sin \varphi \\
0 & -\sin \varphi & \cos \varphi
\end{array}\right)\left(\begin{array}{l}
\mathbf{T}_{n} \\
\mathbf{N}_{n} \\
\mathbf{B}_{n}
\end{array}\right)
$$


with a certain angle $\varphi(s)$. Here, we will call the set $\left\{\mathbf{T}_{1}, \mathbf{N}_{1}, \mathbf{N}_{2}\right\}$ as Natural rotation-minimizing (NRMF). Therefore, we have the alternative frame equations

$$
\left(\begin{array}{l}
\mathbf{T}_{1}^{\prime} \\
\mathbf{N}_{1}^{\prime} \\
\mathbf{N}_{2}^{\prime}
\end{array}\right)=\left(\begin{array}{ccc}
0 & \kappa_{1}(s) & \kappa_{2}(s) \\
-\kappa_{1}(s) & 0 & 0 \\
-\kappa_{2}(s) & 0 & 0
\end{array}\right)\left(\begin{array}{l}
\mathbf{T}_{1} \\
\mathbf{N}_{1} \\
\mathbf{N}_{2}
\end{array}\right),
$$

where

$$
\left.\begin{array}{l}
\kappa_{1}=\kappa_{n} \cos \varphi, \kappa_{2}=\kappa_{n} \sin \varphi, \\
\varphi=\tan ^{-1}\left(\frac{\kappa_{2}}{\kappa_{1}}\right) ; \kappa_{1} \neq 0, \\
\sqrt{\kappa_{1}^{2}+\kappa_{2}^{2}}=\kappa, \varphi-\varphi_{0}=-s_{0} \int \tau_{n} d s .
\end{array}\right\}
$$

We denote a surface $M$ in $E^{3}$ by

$$
\begin{gathered}
\mathbf{Q}(s, \theta)=\left(x_{1}(s, \theta), x_{2}(s, \theta), x_{3}(s, \theta)\right), \\
(s, \theta) \in D \subseteq R^{2} .
\end{gathered}
$$

Let $\mathbf{U}$ be the standard unit normal vector field on a surface $M$ defined by $\mathbf{U}=\frac{\mathbf{Q}_{s} \times \mathbf{Q}_{\theta}}{\left\|\mathbf{P}_{s} \times \mathbf{P}_{\theta}\right\|}$, where, $\mathbf{Q}_{i}=\frac{\partial \mathbf{Q}}{\partial i}$. Then the metric (first fundamental form) $I$ of a surface $M$ is defined by

$$
I=g_{11} d s^{2}+2 g_{12} d s d \theta+g_{22} d \theta^{2},
$$

where $g_{11}=<Q_{s}, Q_{s} \quad>, \quad g_{12}=<Q_{s}, Q_{\theta} \quad>$ , $g_{22}=<Q_{\theta}, Q_{\theta}>$. We define the second fundamental form $I I$ of $M$ by

$$
I I=h_{11} d s^{2}+2 h_{12} d s d \theta+h_{22} d \theta^{2},
$$

where $h_{11}=<Q_{s s}, \mathbf{U}>, h_{12}=<Q_{\theta s}, \mathbf{U}>$ , $h_{22}=<Q_{\theta \theta}, \mathbf{U}>$. The Gaussian curvature $K$ is given by

$$
K(s, \theta)=\frac{h_{11} h_{22}-h_{12}^{2}}{g_{11} g_{22}-g_{12}^{2}} .
$$

\section{Sweeping surfaces}

In this section, we first define a sweeping surface in Euclidean 3 -space $E^{3}$. Let $\beta(s)$ be the Natural mate curve of a unit speed curve $\alpha(s)$, and $\left\{\mathbf{T}_{1}, \mathbf{N}_{1}, \mathbf{N}_{2}\right\}$ be the NRMF along $\beta(s)$. Then a parametrization of a sweeping surface is given by

$$
\begin{aligned}
& M: \mathbf{Q}(s, \theta)=\beta(s)+T(s) \mathbf{x}(\theta) \\
= & \beta(s)+\cos \theta \mathbf{N}_{1}(s)+\sin \theta \mathbf{N}_{2}(s) .
\end{aligned}
$$

For such a sweeping surface, $\beta(s)$ and $\mathbf{x}(\theta)$ are called the spine curve and the profile curve, respectively; $\mathbf{x}(\theta)=(0, \cos \theta, \sin \theta)^{t}$ is with another parameter $\theta \in I \subseteq R$. The special orthogonal matrix $T(s)=\left\{\mathbf{T}_{1}, \mathbf{N}_{1}, \mathbf{N}_{2}\right\}$ specifies the NRMF along $\beta(s)$. From now on, we shall often not write the parameters $s$, and $\theta$ explicitly in our formulae. By using Eq. (7), we can obtain that

$$
\left.\begin{array}{l}
\mathbf{Q}_{s}(s, \theta)=\left(1-\kappa_{1} \cos \theta-\kappa_{2} \sin \theta\right) \mathbf{T}_{1}, \\
\mathbf{Q}_{\theta}(s, \theta)=-\sin \theta \mathbf{N}_{1}+\cos \theta \mathbf{N}_{2},
\end{array}\right\}
$$

and

$$
\left.\begin{array}{l}
g_{11}=\left(1-\kappa_{1} \cos \theta-\kappa_{2} \sin \theta\right)^{2}, \\
g_{12}=0, g_{22}=1
\end{array}\right\}
$$

The unit normal vector is

$$
\mathbf{U}(s, \theta)=\cos \theta \mathbf{N}_{1}+\sin \theta \mathbf{N}_{2} .
$$

It is clear that $\mathbf{U}(s, \theta)$ is contained in the normal plane of the spine curve $\beta(s)$, because it is perpendicular to $\mathbf{T}_{1}$. Also,

$\gamma(\theta)=\mathbf{Q}\left(\theta, s_{0}\right)=\alpha\left(s_{0}\right)+\cos \theta \mathbf{N}_{1}\left(s_{0}\right)+\sin \theta \mathbf{N}_{2}\left(s_{0}\right)$,

is a planar unit speed curve. The unit tangent vector to $\gamma(\theta)$ is

$$
\mathbf{T}_{\gamma}(\theta)=-\sin \theta \mathbf{N}_{1}\left(s_{0}\right)+\cos \theta \mathbf{N}_{2}\left(s_{0}\right),
$$

and thus the unit principal normal vector of $\gamma$ is given by

$$
\begin{gathered}
\mathbf{N}_{\gamma}=\mathbf{e}_{1}\left(s_{0}\right) \times \mathbf{T}_{\gamma}(\theta)=-\cos \theta \mathbf{N}_{1}\left(s_{0}\right)-\sin \theta \mathbf{N}_{2}\left(s_{0}\right) \\
=\mathbf{U}\left(s_{0}, \theta\right)
\end{gathered}
$$

Thus, the surface normal $\mathbf{U}\left(s_{0}, \theta\right)$ is identical to the principal normal $\mathbf{N}_{\gamma}$, that is, the curve $\gamma(\theta)$ is a geodesic curve on $\mathbf{Q}\left(\theta, s_{0}\right)$. Subsequently, the $\theta$-parameter curve cannot be asymptotic curve. By simple computations, we have:

$$
\begin{aligned}
& \mathbf{Q}_{s s}=-\left(\kappa_{1}^{\prime} \sin \theta+\kappa_{2}^{\prime} \cos \theta\right) \mathbf{T}_{1} \\
& +\left(1-\kappa_{1} \sin \theta-\kappa_{2} \cos \theta\right)\left(\kappa_{1} \mathbf{N}_{1}+\kappa_{2} \mathbf{N}_{2}\right) \\
& \mathbf{Q}_{s \theta}=-\left(\kappa_{1} \cos \theta-\kappa_{2} \sin \theta\right) \mathbf{T}_{1} \\
& \mathbf{Q}_{\theta \theta}=-\left(\sin \theta \mathbf{N}_{1}+\cos \theta \mathbf{N}_{2}\right)
\end{aligned}
$$

This leads to $h_{11}, h_{12}$, and $h_{22}$ are given by:

$$
\left.\begin{array}{l}
h_{11}=\left(1-\kappa_{1} \sin \theta-\kappa_{2} \cos \theta\right)\left(\kappa_{1} \sin \theta+\kappa_{2} \cos \theta\right), \\
h_{12}=0, h_{22}=1 .
\end{array}\right\}
$$

Proposition 3.1. Let $M$ be the sweeping surface as in Eq. (12). Then:

(1) The $s$-parameter curve is a geodesic on $M$ if and only if

$$
\left.\begin{array}{c}
\kappa_{1} \sin \theta-\kappa_{2} \cos \theta+\kappa_{1} \kappa_{2} \cos \theta+\frac{1}{2}\left(\kappa_{2}^{2}-\kappa_{1}^{2}\right) \sin 2 \theta=0, \\
\kappa_{1}^{\prime} \cos \theta+\kappa_{2}^{\prime} \sin \theta=0
\end{array}\right\}
$$


(2) The $s$-parameter curve is an asymptotic curve on $M$ if and only if.

$$
\theta=\tan ^{-1}\left(\frac{\kappa_{2} \pm \kappa_{1} \sqrt{\left|\kappa_{2}^{2}+\kappa_{1}^{2}-1\right|}}{\kappa_{1} \mp \kappa_{2} \sqrt{\left|\kappa_{2}^{2}+\kappa_{1}^{2}-1\right|}}\right) .
$$

Proof. Form Eqs. (15), and (16), we have:

(1) The $s$-parameter curve is a geodesic if and only if $\mathbf{Q}_{s s} \times \mathbf{U}(s, \theta)=\mathbf{0}$, that is,

$$
\left[\kappa_{1} \sin \theta-\kappa_{2} \cos \theta+\kappa_{1} \kappa_{2} \cos \theta\right.
$$$$
+\frac{1}{2}\left(\kappa_{2}^{2}-\kappa_{1}^{2}\right) \sin 2 \theta \mathbf{e}_{1}
$$$$
\left.+\left[\kappa_{1}^{\prime} \cos \theta+\kappa_{2}^{\prime} \sin \theta\right]\left(\sin \theta \mathbf{N}_{1}-\cos \theta \mathbf{N}_{2}\right)=0 .\right\}
$$

Since $\mathbf{T}_{1}, \mathbf{N}_{1}$ and $\mathbf{N}_{2}$ are linearly independent unit vectors, we have the equation system (19).

(2) The $s$-parameter curve is an asymptotic curve on $M$ if and only if $<\mathbf{U}, \mathbf{Q}_{s s}>=0$, that is,

$$
\left(1-\kappa_{1} \sin \theta-\kappa_{2} \cos \theta\right)\left(\kappa_{1} \cos \theta+\kappa_{2} \sin \vartheta\right)=0,
$$

from which it follows that

$$
\sin \theta=\frac{\kappa_{2} \pm \kappa_{1} \sqrt{\left|\kappa_{2}^{2}+\kappa_{1}^{2}-1\right|}}{\kappa_{2}^{2}+\kappa_{1}^{2}}
$$

and

$$
\cos \theta=\frac{\kappa_{1} \mp \kappa_{2} \sqrt{\left|\kappa_{2}^{2}+\kappa_{1}^{2}-1\right|}}{\kappa_{2}^{2}+\kappa_{1}^{2}},
$$

as claimed.

\subsection{Singularities and lines of curvature}

WSingularities and lines of curvature are essential for understanding the properties of sweeping surfaces and are investigated in the following: $M$ has singular points if and only if the first derivatives are linearly dependent, that is,

$$
\mathbf{Q}_{\theta} \times \mathbf{Q}_{s}=\left(1-\kappa_{1} \cos \theta-\kappa_{2} \sin \theta\right) \mathbf{U}=\mathbf{0} .
$$

Since $\mathbf{U}$ is a nonzero unit vector, then $1-$ $\kappa_{1} \cos \theta-\kappa_{2} \sin \theta=0$; hence,

$$
\sin \theta=\frac{\kappa_{2} \pm \kappa_{1} \sqrt{\left|\kappa_{2}^{2}+\kappa_{1}^{2}-1\right|}}{\kappa_{2}^{2}+\kappa_{1}^{2}}
$$

, and

$$
\cos \theta=\frac{\kappa_{1} \mp \kappa_{2} \sqrt{\left|\kappa_{2}^{2}+\kappa_{1}^{2}-1\right|}}{\kappa_{2}^{2}+\kappa_{1}^{2}} .
$$

Hence there are two sets of singular points on $M$. From Eq. (12) it follows that the expression of these two sets is

$$
\begin{aligned}
\mathbf{r}_{ \pm}(s)= & \beta(s)+\frac{\kappa_{1} \mp \kappa_{2} \sqrt{\left|\kappa_{2}^{2}+\kappa_{1}^{2}-1\right|}}{\kappa_{2}^{2}+\kappa_{1}^{2}} \mathbf{N}_{1}(s) \\
& +\frac{\kappa_{2} \pm \kappa_{1} \sqrt{\left|\kappa_{2}^{2}+\kappa_{1}^{2}-1\right|}}{\kappa_{2}^{2}+\kappa_{1}^{2}} \mathbf{N}_{2}(s) .
\end{aligned}
$$

From Eqs. (14), and (18) it can be found that $g_{12}=h_{12}=0$. Hence, the $\vartheta$-and $s$ curves of $M$ are lines of curvature. Surfaces whose parametric curves are lines of curvature have several applications in geometric design [2]. In the case of sweeping surfaces, one has to compute the offset surfaces $\mathbf{Q}_{f}(s, \theta)=\mathbf{Q}(s, \theta)+\rho \mathbf{U}(s, \theta)$ of a given surface $\mathbf{Q}(s, \theta)$ at a certain distance $\rho$. Therefore, the offsetting operation for sweeping surface can be reduced to the offsetting of planar profile curve, which is much easier to deal with. Hence, we can state the following proposition:

Proposition 3.2. Consider a sweeping surface $M$ as in Eq. (12). Let $\mathbf{x}_{f}(\theta)$ be the planar offset of the profile $\mathbf{x}(\theta)$ at distance $\rho$. Then the offset surface $\mathbf{Q}_{f}(s, \theta)$ is again a sweeping surface, generated by the spine curve $\beta(s)$ and profile curve $\mathbf{x}_{f}(\theta)$.

In order to study the shape of $M$ we examine the Gaussian curvature $K(s, \theta)=\chi_{1} \chi_{2}$. Here, the $\chi_{i}(s, \theta)(i=1,2)$ are the principal curvatures of the sweeping surface. For this purpose, the value of one principal curvature is

$$
\chi_{1}:=\left\|\frac{d \mathbf{x}}{d \theta} \times \frac{d^{2} \mathbf{x}}{d \theta^{2}}\right\|\left\|\frac{d \mathbf{x}}{d \theta}\right\|^{-3}=1 .
$$

And, the curvature of the $s$-parameter curve $(\theta-$ constant) is

$$
\chi\left(s, \theta_{0}\right):=\frac{\left\|\mathbf{Q}_{s} \times \mathbf{Q}_{s s}\right\|}{\left\|\mathbf{Q}_{s}\right\|^{3}}=\frac{\kappa_{n}}{1-\kappa_{1} \cos \theta-\kappa_{2} \sin \theta} .
$$

Moreover, the principal curvature $\chi_{2}$ is related to the curvature $\chi(s, \theta)$ via Meusnier's Theorem [1]:

$$
\chi_{2}=\chi(s, \theta) \cos \Gamma
$$

where $\Gamma=\cos ^{-1}<\mathbf{U}, \mathbf{N}_{n}>$. Hence, the Gaussian curvature $K(s, \theta)$ can be rewritten as

$$
K(s, \theta)=\chi(s, \theta) \cos \Gamma .
$$

In CAGD, conditions that guarantee the convexity or curves which produce parabolic points of a surface are required in various applications (such 
as manufacturing of sculptured surfaces, or layered manufacturing). However, for the sweeping surface $M$ the convexity can be controlled with the help of the geometrical properties as:

$$
K(s, \theta)=0 \Leftrightarrow \chi(s, \theta) \cos \Gamma=0 .
$$

There are two possible cases:

Case (1) occurs when $\chi(s, \theta)=0$. From Eq. (25) it can be found that if $\kappa_{n}(s)=0$, then $\chi=0$. This means that the spine curve $\beta(s)$ is degenerate into a straight line. Therefore, an inflection or flat point of the spine curve generates a parabolic curve $\theta=$ const. on the sweeping surface.

Case (2) occurs when $\Gamma=\pi / 2$. This means that if $\mathbf{U}(s, \theta) \| \mathbf{B}_{n}$, hence $\cos \Gamma=0$. Then the curve $\beta(s)$ is not only a line of curvature but also asymptotic of the sweeping surface.

Corollary 3.1. Consider a sweeping surface $M$ with spine and profile curves have non-vanishing curvatures everywhere. If the normal $\mathbf{U}(s, \theta)$ is never parallel to the principal normal $\mathbf{N}_{n}(s)$ of the spine curve $\beta(s)$, then $M$ has no parabolic points.

According to Proposition 3.1, and Eq. (12) the expression of the two parabolic curves is $\mathrm{x}(\mathrm{s})=\beta(s)+\frac{\kappa_{1} \mp \kappa_{2} \sqrt{\left|\kappa_{2}^{2}+\kappa_{1}^{2}-1\right|}}{\kappa_{2}^{2}+\kappa_{1}^{2}} \mathbf{N}_{1}$

$+\frac{\kappa_{2} \pm \kappa_{1} \sqrt{\left|\kappa_{2}^{2}+\kappa_{1}^{2}-1\right|}}{\kappa_{2}^{2}+\kappa_{1}^{2}} \mathbf{N}_{2}$.

Corollary 3.2. Let $M$ be a sweeping surface with spine and profile curves have non-vanishing curvatures everywhere. Then $M$ has exactly two parabolic curves if and only if the spine curve $\beta(s)$ is an asymptotic curve.

\subsection{Developable surfaces}

We now will discuss in what conditions the sweeping surfaces are developable surfaces. Therefore, we analyze the case, that the profile curve $\mathbf{x}$ degenerates into a straight line, thus generating a developable surface

$$
F: \mathbf{Q}(s, u)=\beta(s)+u \mathbf{N}_{2}(s), u \in R .
$$

Similarly, from Eq. (12), we have the following developable surface

$$
F^{\perp}: \mathbf{Q}^{\perp}(s, u)=\beta(s)+u \mathbf{N}_{1}(s), u \in R .
$$

It is possible to show $\mathbf{Q}(s, 0)=\beta(s)$ (resp. $\left.\mathbf{Q}^{\perp}(s, 0)=\beta(s)\right), 0 \leq s \leq L$, that is, the surface $F$ (resp. $F^{\perp}$ ) interpolates the curve $\beta(s)$. Furthermore, since

$$
\mathbf{Q}_{s} \times \mathbf{Q}_{u}:=-\left(1-u \kappa_{2}\right) \mathbf{N}_{1}(s),
$$

then $F^{\perp}$ is the normal developable surface of $F$ along $\beta(s)$. Therefore, the surface $F$ (resp. $F^{\perp}$ ) interpolates the curve $\beta(s)$, and $\beta(s)$ is a line of curvature of $F$ (resp. $F^{\perp}$ ).

Theorem 3.1. (Existence and uniqueness). Under the above notations there exists a unique developable surface expressed by Eq. (29).

Proof. For the existence, we have the developable expressed by Eq. (29). On the other hand, since $F$ is a ruled surface, we assume that

$$
\left.\begin{array}{l}
F: \mathbf{Q}(s, u)=\beta(s)+u \mathbf{a}(s), u \in R, \\
\mathbf{a}(s)=a_{1}(s) \mathbf{T}_{1}+a_{2}(s) \mathbf{N}_{1}+a_{3}(s) \mathbf{N}_{2}, \\
\|\mathbf{a}(s)\|^{2}=a_{1}^{2}+a_{2}^{2}+a_{3}^{2}=1, \mathbf{a}^{\prime}(s) \neq \mathbf{0} .
\end{array}\right\}
$$

It can be immediately seen that $F$ is developable if and only if

$\operatorname{det}\left(\beta^{\prime}, \mathbf{a}, \mathbf{a}^{\prime}\right)=0 \Leftrightarrow a_{2}\left(a_{3}^{\prime}+a_{1} \kappa_{2}\right)-a_{3}\left(a_{2}^{\prime}+a_{1} \kappa_{1}\right)=0$.

On the other hand, in view of Eq. (3.21), we have

$$
\left(\mathbf{Q}_{s} \times \mathbf{Q}_{u}\right)(s, u)= \pm \lambda(s, u) \mathbf{N}_{1}
$$

where $\lambda=\lambda(s, u)$ is a differentiable function. Further, the normal vector $\mathbf{Q}_{s} \times \mathbf{Q}_{v}$ at the point $(s, 0)$ is

$$
\left(\mathbf{Q}_{s} \times \mathbf{Q}_{u}\right)(s, 0)=-a_{3} \mathbf{N}_{1}+a_{2} \mathbf{N}_{2} .
$$

Thus, from Eqs. (34), and (35), one finds that:

$$
a_{2}=0, \text { and }_{3}=\lambda(s, 0),
$$

which follows from Eq. (35) that $a_{3} a_{1} \kappa_{1}=0$, which leads to $a_{1} a_{3}=0$, with $\kappa_{1} \neq 0$. If $(s, 0)$ is a regular point (i.e., $\lambda(s, 0) \neq 0$ ), then $a_{3}(s) \neq 0$, and $a_{1}=0$. Therefore, the direction of $\mathbf{a}(s)$ is equal to the direction of $\mathbf{e}_{3}(s)$. This means that uniqueness holds .

In similar arguments for $F^{\perp}$, we can give the corresponding Theorem of Theorem 3.1. (Existence and uniqueness) we omit the details here. Thus, the Joachimsthal theorem can be stated as the following:

Theorem 3.2. (Joachimsthal). Let $F$ and $F^{\perp}$ two developable surfaces such that $F \cap F^{\perp}=\beta(s)$ is a regular curve and $\left\langle\mathbf{N}_{1}, \mathbf{N}_{2}\right\rangle=0$ along $\beta(s)$, where $\mathbf{N}_{1}$ and $\mathbf{N}_{2}$ are unitary normal vector fields to $F$ and $F^{\perp}$, respectively. Then $\beta(s)$ is a line of curvature of $F$ if and only if it is a line of curvature of $F^{\perp}$. 
In above theorems, we not only prove the existence and uniqueness of the developable surface, but also give the concrete expression of the surface. This is very meaningful in practical application. As it is will known, there are three types of developable surfaces, the given curve can be classified into three kinds correspondingly. In what follows, we will discuss the relationship between the given curve $\beta(s)$ and its isoparametric developable. The first case is when,

$$
\mathbf{N}_{2} \times \mathbf{N}_{2}^{\prime}=\mathbf{0} \Leftrightarrow \kappa_{n} \sin \varphi \mathbf{N}_{1}=\mathbf{0} .
$$

In this case, $F$ is referred to as a cylindrical surface. Since $\mathbf{N}_{1}$ is a nonzero unit vector, then the $F$ is a cylindrical surface if and only if $\sin \varphi=$ $0 \Leftrightarrow \varphi=0$ or $\pi$. However, in any case, we have $\varphi^{\prime}=0$, then $\tau_{n}=0$. That is, the curve $\beta$ is a planar curve, and $F$ is a binormal surface. By similar argument, we can also have the following:

$$
\mathbf{N}_{2} \times \mathbf{N}_{2}^{\prime} \neq \mathbf{0}
$$

This implies that the $F$ is a non-cylindrical surface. Therefore, the first derivative of the directrix is

$$
\beta^{\prime}(s)=\mathbf{C}^{\prime}(s)+\sigma(s) \mathbf{N}_{2}^{\prime}(s)+\sigma^{\prime}(s) \mathbf{N}_{2}(s),
$$

where $\mathbf{C}^{\prime}$ is the first derivative of the striction curve, $\sigma(s)$ is a smooth function. Therefore, we obtain

$$
<\mathbf{N}_{2} \times \mathbf{N}_{2}^{\prime}, \mathbf{C}^{\prime}>=0 .
$$

Similarly, there are two possible cases which satisfy Eq. (40), as presented in the following: The first case is when the first derivative of the striction curve is $\mathbf{C}^{\prime}=0$. Geometrically this condition implies that the striction curve degenerates to a point, and the ruled surface becomes a cone; the striction point of a cone is commonly referred to as the vertex. Therefore, the surface $F$ is a cone if and only if there exists a fixed point $\mathbf{C}$ and a function $\sigma(s)$ such that $\sigma \kappa_{n} \sin \varphi=1, \sigma^{\prime}=0$, which imply that

$$
\sigma=\text { const. }=\frac{1}{\kappa_{n} \sin \varphi} \Leftrightarrow \kappa_{n} \sin \varphi=\kappa_{n 0} \sin \varphi_{0},
$$

where $\varphi_{0}=\varphi(0)$, and $\kappa_{n 0}=\kappa_{n}(0)$. In Eq. (41), $\kappa_{n} \sin \varphi$ is a constant. However, if $\varphi$ is constant, then $\tau_{n}=0$, that is, $\beta(s)$ is a planar line of curvature, and $\kappa_{n}$ is also constant. Similarly, if $\kappa_{n}$ is a constant, we can have $\tau_{n}=0$, and $\varphi$ is a constant. Then the curve $\beta(s)$ is the arc of a circle.

The second case is when $\kappa_{n} \sin \varphi \neq \kappa_{n 0} \sin \varphi_{0}$, we have $\mathbf{C}^{\prime} \neq \mathbf{0}$. Since $<\mathbf{N}_{2} \times \mathbf{N}_{2}^{\prime}, \mathbf{C}^{\prime}>=0$, $<\mathbf{N}_{2}, \mathbf{N}_{2}^{\prime}>=0$, and the condition for $\mathbf{C}$ to be striction curve is equivalent to $\left\langle\mathbf{C}^{\prime}, \mathbf{N}_{2}\right\rangle=0$ we can get $\mathbf{C}^{\prime} \| \mathbf{N}_{2}$. This means the tangent surface is composed of tangents of a spatial curve, the cuspidal edge $\beta(s)$.

\subsection{Examples}

In what follows, we will discuss the construction of developable surfaces with the given curve as a line of curvature.

Example 1. Given the cylindrical helix

$$
\alpha(s)=\frac{1}{\sqrt{2}}(-\cos s,-\sin s, s), \quad 0 \leq s \leq 2 \pi .
$$

Then, it is easy to show that:

$$
\left.\begin{array}{l}
\mathbf{T}(s)=\frac{1}{\sqrt{2}}(\sin s,-\cos s, 1), \\
\mathbf{N}(s)=(\cos s, \sin s, 0), \\
\mathbf{B}(s)=\frac{1}{\sqrt{2}}(-\sin s, \cos s, 1), \\
\kappa=\tau=\frac{1}{\sqrt{2}} .
\end{array}\right\}
$$

According to Eq. (2), the SerretFrenet frame of $\beta(s)$ can be described as $\mathbf{T}_{n}(s)=(\cos s, \sin s, 0)$ $\mathbf{N}_{n}(s)=(-\sin s, \cos s, 0)$ $\mathbf{B}_{n}(s)=(0,0,1)$, $\kappa_{n}=1$, and $\tau_{n}=0$.

From $\tau_{n}=0$, we find $\varphi(s)=\varphi_{0}$ is a constant, and Eq. (41) is satisfied. If $\varphi_{0}=0$ or $\pi$ the developable surface is a cylinder. Choose $\varphi_{0}=0$ for example, the corresponding surface is expressed as

$$
F: \mathbf{Q}(s, u)=(-\sin s, \cos s, u)
$$

which is shown in Fig. 1 If we choose $\varphi_{0}=\frac{\pi}{4}$, then

$\mathbf{N}_{2}(s)=-\sin \varphi \mathbf{N}_{n}+\cos \varphi \mathbf{B}_{n}=\frac{1}{\sqrt{2}}(-\sin s, \cos s, 1)$,

and the developable surface

$\mathrm{F}: \mathrm{Q}(\mathrm{s}, \mathrm{u})=\left(-\sin s-\frac{u}{\sqrt{2}} \sin s, \cos s+\frac{u}{\sqrt{2}} \cos s, \frac{u}{\sqrt{2}}\right)$, is a cone possesses $\beta(s)$ as a line of curvature (See Fig. 2). 


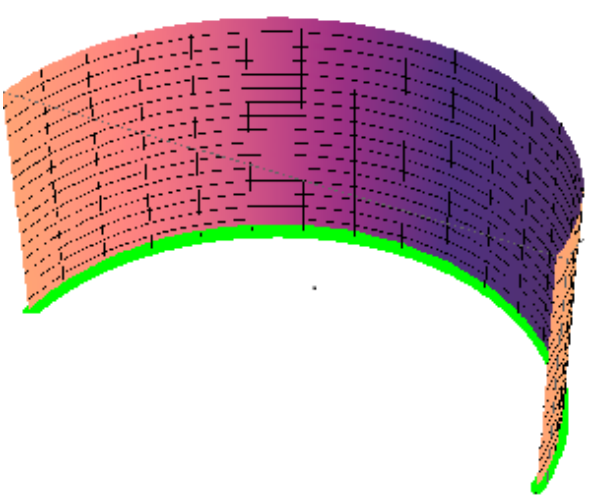

Figure 1: Cylinder with $0 \leq s \leq \pi$ and $0 \leq$ $u \leq 1$.

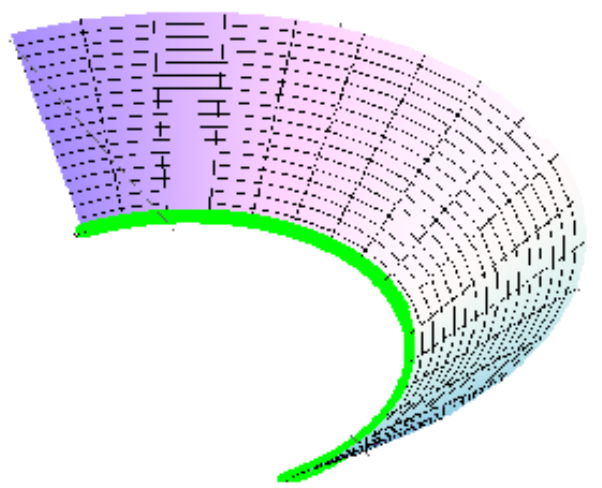

Figure 2: Cone with $0 \leq s \leq \pi$ and $0 \leq u \leq 1$

Example 2. Given the slant helix $\alpha(s)=\left(\frac{3}{4} \cos s+\frac{\cos 3 s}{12}, \frac{3}{4} \sin s+\frac{\sin 3 s}{12},-\frac{\sqrt{3}}{2} \cos s\right)$. After simple computation, we have:

$$
\begin{gathered}
\mathbf{T}(s)=\left(-\frac{3}{4} \sin s-\frac{\sin 3 s}{4}, \frac{3}{4} \cos s+\frac{\cos 3 s}{4}, \frac{\sqrt{3}}{2} \sin s\right), \\
\mathbf{N}(s)=\left(-\frac{\sqrt{3}}{2} \cos 2 s,-\frac{\sqrt{3}}{2} \sin 2 s, \frac{1}{2}\right), \\
\mathbf{B}(s)=\left(\frac{3 \cos -\cos 3 s}{4}+\frac{\cos 3 s}{12}, \sin ^{3} s, \frac{\sqrt{3}}{2} \cos s\right) \\
\kappa(s)=\sqrt{3} \cos s, \tau(s)=-\sqrt{3} \sin s .
\end{gathered}
$$

Furthermore, the Serret-Frenet frame of $\beta(s)$ can be described as: $\left.\begin{array}{l}\mathbf{T}_{n}(s)=\left(-\frac{\sqrt{3}}{2} \cos 2 s,-\frac{\sqrt{3}}{2} \sin 2 s, \frac{1}{2}\right), \\ \mathbf{N}_{n}(s)=\left(\frac{\sin 4 s}{4}, \frac{-3-\cos 4 s}{4},-\frac{\sqrt{3}}{2} \sin 2 s\right), \\ \mathbf{B}_{n}(s)=\left(\frac{3-\cos 4 s}{4}, \frac{-\cos -\cos 3 s}{2}, \frac{\sqrt{3}}{2} \cos 2 s\right), \\ \kappa_{n}=\sqrt{3}, \text { and } \tau_{n}=-1 .\end{array}\right\}$

It is easy to show that $\varphi(s)=s+\varphi_{0}$. If we choose $\varphi_{0}=0$, then $\varphi(s)=s$, and $\kappa \sin \varphi \neq \kappa_{0} \sin \varphi_{0}$. Thus, the corresponding surface is a tangential surface. By computing
$\left(\begin{array}{l}\mathbf{T}_{1} \\ \mathbf{N}_{1} \\ \mathbf{N}_{2}\end{array}\right)=\left(\begin{array}{lll}1 & 0 & 0 \\ 0 & \cos s & \sin s \\ 0 & -\sin s & \cos s\end{array}\right)\left(\begin{array}{l}\mathbf{T}_{n} \\ \mathbf{N}_{n} \\ \mathbf{B}_{n}\end{array}\right)$ the surface is expressed as

$$
\begin{aligned}
F: & \mathbf{Q}(s, u)=\left(\frac{\sin 4 s}{4}, \frac{-3-\cos 4 s}{4},-\frac{\sqrt{3}}{2} \sin 2 s\right) \\
+ & u\left(-\sin s \mathbf{N}_{n}+\cos s \mathbf{B}_{n}\right) \\
= & \left(\frac{\sin 4 s}{4}, \frac{-3-\cos 4 s}{4},-\frac{\sqrt{3}}{2} \sin 2 s\right)+ \\
& u(0,-\sin s, \cos s) \\
& \left(\begin{array}{ccc}
-\frac{\sqrt{3}}{2} \cos 2 s & -\frac{\sqrt{3}}{2} \sin 2 s \\
\frac{\sin 4 s}{4} & \frac{-3-\cos 4 s}{4} & -\frac{\sqrt{3}}{2} \sin 2 s \\
\frac{3 \cos -\cos 3 s}{4}+\frac{\cos 3 s}{12} & \sin ^{3} s & \frac{\sqrt{3}}{2} \cos s
\end{array}\right),
\end{aligned}
$$

which is shown in Fig. 3; $0 \leq s \leq \pi / 4$, and $0 \leq u \leq 3$.

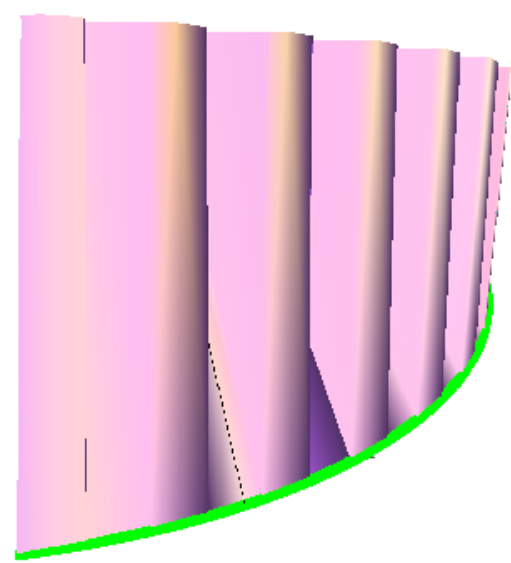

Figure 3: Tangential surface with Natural mate of slant helix.

\section{Conclusion}

This paper investigate the properties of sweeping surface by setting up an orthonormal NRMF to each point of the Naturam mate curve of a space curve. Consequently, we have solved the problem of requiring the surface that is sweeping surface and at the same time developable surface. Moreover, examples illustrates the application of the obtained formula are introduced. There are several opportunities for further work. The methodology used here can be applied to the sweeping surface in different spaces such as LorentzMinkowski space, isotropic space, and etc.

\section{References:}

[1] MP. do Carmo. Differential Geometry of Curves and Surface, Prentice-Hall, Englewood Cliffs, NJ, (1976). 
[2] W. Lu, and H. Pottmann. Pipe surfaces with rational spine curve are rational, Comput. Aided Geom. Des., 13 (1996), pp. 621-628.

[3] A. Stasiak, JH. Maddocks. Best Packing in Proteins and DNA, Nature London , 406 (2000), pp. 251-253.

[4] A. Maritan, C. Micheletti, A. Trovato, J.R Banavar. Optimal shapes of compact strings," Nature London , 406 (2000), pp. 287-290.

[5] SN. Krivoshapko, and C.A. Hyeng. Classi cation of cyclic surfaces and geometrical research of canal surfaces, Int. J. Res. Rev. Appl. Sci. 12 (2012), No. 3, PP. 360-374.

[6] YH Kim, H. Liu, and J. Qian. Some Characterizations of canal surfaces, Bull. Korean Math. Soc. 53 (2016), No. 2, pp. 461-477.

[7] JH Qian, and YH. Kim. Some Classification of canal surfaces with the Gauss map, Bull. Malays. Math. Sci. Soc, 42: 3261-3272, 2019.

[8] M.A. Soliman, W.M. Mahmoud, E.M. Solouma, and M. Bary. The new study of some characterization of canal surfaces with Weingarten and linear Weingarten types according to Bishop frame, J. of the Egyptian Math. Soci., (2019) 27-26.

[9] H. Pottmann, and J. Wallner. Computational Line Geometry, Springer-Verlag, Berlin, Heidelberg, (2001).

[10] L. Cui, D. L Wang, and J.S. Dai. Kinematic geometry of circular surfaces with a fixed radius based on Euclidean invariants," ASME J. Mech. 101009-2 / Vol. 131, October 2009.

[11] W. Wang, and B. Joe. Robust computation of the rotation minimizing frame for sweep surface modelling, Computer-Aided Design, 29: 379-91, 1997.

[12] W. Wang, B. Jüttler, D. Zheng, andY. Liu. Computation of rotating minimizing frames, ACM Transactions on Graphics, 27: 1-18, 2008 .

[13] RA. Abdel-Baky. Developable surfaces through sweeping surfaces, Bulletin of the Iranian Mathematical Society, August, Volume 45, Issue 4, 951-963, 2019.

[14] H. Pottmann, and G. Farin Developable Rational Bezier and B-Spline durfaces, Computer-Aided Geometric Design, 12, 513531, 1995.
[15] HY. Zhao, and GJ. Wang. A new method for designing a developable surface utilizing the surface pencil through a given curve, Progress in Nature Science, 18: 105-110, 2008.

[16] CY. Li, RH. Wang, and CG. Zhu. An approach for designing a developable surface through a given line of curvature, ComputerAided Design 45, 621-627, 2013.

[17] RL. Bishop. There is more than one way to frame a curve, Amer. Math. Monthly 82, 246251, 1975.

[18] F. Klok. Two moving coordinate frames for sweeping along a 3D trajectory,. Comput. Aided Geom. Design 3, 217-229, 1986.

[19] RT. Farouki, C. Giannelli , and ML. Sampoli. Rotation-minimizing osculating frames, Comput Aided Geom Des 31(1): 27-4, 2011.

[20] D. Sharief, YC. Bang, and A. Azeb. Natural mates of Frenet curves in Euclidean 3-space, Turk. J. Math. 42 2826-2840, 2018.

\section{Contribution of individual authors to the creation of a scientific article (ghostwriting policy)}

Author Contributions: Please, indicate the role and the contribution of each author:

Example

John Smith, Donald Smith carried out the simulation and the optimization.

George Smith has implemented the Algorithm 1.1 and 1.2 in $\mathrm{C}++$.

Maria Ivanova has organized and executed the experiments of Section 4.

George Nikolov was responsible for the Statistics.

Follow: www.wseas.org/multimedia/contributorrole-instruction.pdf

\section{Sources of funding for research presented in a scientific article or scientific article itself}

Report potential sources of funding if there is any

\section{Creative Commons Attribution License 4.0 (Attribution 4.0 International, CC BY 4.0)}

This article is published under the terms of the Creative Commons Attribution License 4.0

https://creativecommons.org/licenses/by/4.0/deed.en US 